\title{
Six-month results of a repositionable and retrievable pericardial valve for transcatheter aortic valve replacement: The Direct Flow Medical aortic valve
}

\author{
Hendrik Treede, MD, ${ }^{\mathrm{a}}$ Thilo Tübler, MD, ${ }^{\mathrm{b}}$ Hermann Reichenspurner, MD, PhD, ${ }^{\mathrm{a}}$ Eberhard Grube, MD, ${ }^{\mathrm{c}}$ \\ Andrea Pascotto, MD, ${ }^{\mathrm{b}}$ Olaf Franzen, MD, ${ }^{\mathrm{a}}$ Ralf Mueller, MD,${ }^{\mathrm{c}}$ Reginald Low, MD, ${ }^{\mathrm{d}}$ \\ Steven F. Bolling, MD, ${ }^{\mathrm{e}}$ Thomas Meinertz, MD, ${ }^{\mathrm{a}}$ and Joachim Schofer, $\mathrm{MD}^{\mathrm{b}}$
}

Objective: Transcatheter aortic valve implantation is considered an alternative for patients at high risk for conventional surgery. The Direct Flow Medical aortic valve (Direct Flow Medical, Inc, Santa Rosa, Calif) is a nonmetallic tissue valve prosthesis intended to treat patients with severe aortic stenosis at high risk for surgery.

Methods: Thirty-one patients at high surgical risk were enrolled in the trial (logistic EuroSCORE $28 \% \pm 7 \%$, Society of Thoracic Surgeons score $23 \% \pm 9 \%$ ). Twenty-two patients underwent successful retrograde transcatheter aortic valve implantation, and 9 patients did not undergo implantation owing to excessive calcifications or access issues. Mean preinterventional gradient and effective orifice area were $49 \pm 14 \mathrm{~mm} \mathrm{Hg}$ and $0.54 \pm 0.16 \mathrm{~cm}^{2}$, respectively, and $71 \%$ of patients were in New York Heart Association functional class III.

Results: Mean postprocedural gradient was $14.9 \pm 5.5 \mathrm{~mm} \mathrm{Hg}$ with an effective orifice area of $1.4 \pm 0.31 \mathrm{~cm}^{2}$. Two patients were converted to surgery and 2 patients died after implantation: 1 of myocardial infarction and 1 of congestive heart failure. One patient had a stroke 2 days after the procedure and 3 patients required a pacemaker. At 6 months the mean aortic valve gradient and effective orifice area were $19.8 \mathrm{~mm} \mathrm{Hg}$ and $1.30 \mathrm{~cm}^{2}$, respectively. The majority of patients had no paravalvular leak (58\%) and $42 \%$ had grade $1 / 4$ paravalvular leak; $69 \%$ were in New York Heart Association functional class I and 25\% were in class II. The 3- and 6-month survivals were $87.1 \%$ and $80.6 \%$, respectively ( $4 / 6$ deaths in 31 patients).

Conclusions: Transcatheter aortic valve implantation using the study valve appears safe and results are promising at 6 months. Severe leaflet and left ventricular outflow tract calcification affects procedural outcome; therefore, careful patient selection is crucial. (J Thorac Cardiovasc Surg 2010;140:897-903)

Transcatheter aortic valve implantation (TAVI) has become a considerable alternative for patients at high risk for open surgical treatment. It offers a treatment option for patients whose condition is considered inoperable owing to extensive calcifications of the ascending aorta or in patients of advanced age with significant comorbidities. The Euro Heart Survey, a European registry of 5000 patients in 25 countries, revealed that $31.8 \%$ of patients with symptomatic aortic stenosis did not undergo surgical aortic valve replacement owing to relevant comorbidities or reduced life expectancy. ${ }^{1}$

\footnotetext{
From the Departments of Cardiovascular Surgery and Cardiology, ${ }^{\text {a }}$ University Heart Center Hamburg, Hamburg, Germany; The Medical Care Center Prof. Mathey, ${ }^{b}$ Prof. Schofer, Hamburg University Cardiovascular Center, Hamburg, Germany; the Department of Cardiology/Angiology, ${ }^{\mathrm{c}}$ HELIOS Heart Center Siegburg, Siegburg, Germany; the Division of Cardiovascular Medicine, ${ }^{\mathrm{d}}$ University of California, Davis Medical Center, Sacramento, Calif; and the University of Michigan Cardiovascular Center, ${ }^{\mathrm{e}}$ Ann Arbor, Mich.

This study was sponsored by Direct Flow Medical, Inc, Santa Rosa, Calif.

Disclosures: None.

Received for publication May 7, 2009; revisions received Dec 18, 2009; accepted for publication Jan 10, 2010; available ahead of print April 15, 2010.

Address for reprints: Hendrik Treede, MD, Department of Cardiovascular Surgery, University Heart Center Hamburg, Martinistrasse 52, 20246 Hamburg / Germany (E-mail: treede@uke.de).

0022-5223/\$36.00

Copyright (c) 2010 by The American Association for Thoracic Surgery doi:10.1016/j.jtcvs.2010.01.017
}

There are currently two commercially available TAVI valve types in Europe (Edwards SAPIEN; Edwards Lifesciences, Irvine, Calif; Medtronic CoreValve Revalving System; Medtronic, Inc, Minneapolis, Minn). Both are based on stent technology and offer only limited possibilities of repositioning and no options for retrieval during implantation. Suboptimal placement of the devices may lead to urgent surgical intervention at increased risk.

The Direct Flow Medical aortic valve (Direct Flow Medical, Inc, Santa Rosa, Calif) is the first transfemorally delivered aortic valve prosthesis that is not based on a metallic stent frame technology. It allows for retrieval, repositioning, or sizing exchange before permanent implantation. Rapid pacing is repeatedly used during standard balloon valvuloplasty of the native aortic valve; it is not required for positioning or deploying the prosthesis. The initial results of this feasibility study of the Direct Flow Medical aortic valve were positive, demonstrating safety during the implantation procedure, with significant reduction in mean transvalvular pressure gradients and an increase in aortic valve area after successful implant. ${ }^{2}$

Herein, we report the immediate postprocedure and 6-month follow-up data from the nonrandomized, prospective clinical trial of 31 enrolled patients eligible for 


\begin{tabular}{|c|c|}
\hline \\
\hline \multicolumn{2}{|c|}{$\mathrm{AR}=$ aortic regurgitation } \\
\hline CEC & $=$ clinical events committee \\
\hline \multicolumn{2}{|c|}{ EuroSCORE $=$ European System for Cardiac } \\
\hline & Operative Risk Evaluation \\
\hline IQR & $=$ interquartile range \\
\hline MACCE & $\begin{aligned}= & \text { major adverse cardiovascular and } \\
& \text { cerebral event }\end{aligned}$ \\
\hline NYHA & $=$ New York Heart Association \\
\hline PFLs & $=$ positioning-and-fill lumina \\
\hline TAVI & $\begin{aligned}= & \text { transcatheter aortic valve } \\
& \text { implantation }\end{aligned}$ \\
\hline TEE & $=$ transesophageal echocardiography \\
\hline TTE & $=$ transthoracic echocardiography \\
\hline
\end{tabular}

transcatheter aortic valve replacement with the Direct Flow Medical aortic valve.

\section{MATERIALS AND METHODS Study Valve}

The Direct Flow Medical aortic valve is designed as a trileaflet bovine pericardial tissue valve (Figure 1). It is attached to an inflatable framework with a slightly tapered, conformable polyester fabric cuff. ${ }^{3,4}$ The upper and lower margins of the cuff consist of two independently inflatable rings connected by a tubular system. The upper aortic ring is connected to three evenly distributed detachable positioning and fill lumina (PFLs) that are used to inflate and deflate the valve, as well as to accurately position in the native annulus. The folded valve is loaded into a $22 \mathrm{~F}$ sheath at the end of a $15 \mathrm{~F}$ delivery catheter with an olive-shaped tip designed to seal the distal end of the delivery sheath and to facilitate an atraumatic passage through the aorta. Two of the PFLs enable inflation and deflation of both the aortic and the ventricular rings on the cuff, and all three allow for correct positioning of the valve in the native annulus by pushing and pulling at the proximal ends. The loaded delivery system is advanced over a guide wire placed transvalvularly in the left ventricle. A basket-like nitinol recovery system can be introduced into the delivery sheath to retrieve the valve if required (Figure 2).

\section{Patients}

Between September 2007 and August 2008, 31 patients (15 men and 16 women; mean age $82 \pm 4$ years) were enrolled at two facilities in Germany (25 patients at University Cardiovascular and University Heart Center, Hamburg, and 6 patients at HELIOS Heart Center, Siegburg). All patients had severe aortic valve stenosis and were at high risk for open cardiac surgery, and the vast majority of patients were symptomatic $(97 \%)$. Relevant comorbidities of all patients are listed in Table 1. Surgical risk was evaluated by a logistic European System for Cardiac Operative Risk Evaluation (EuroSCORE $)^{5}$ and considered high by consensus of two independent experienced cardiac surgeons who were not members of the study group. Inclusion criteria were an aortic valve area of $0.8 \mathrm{~cm}^{2}$ or less, a mean transvalvular pressure gradient of $35 \mathrm{~mm} \mathrm{Hg}$ or more, an aortic annulus diameter between 19 and $23 \mathrm{~mm}$, age of 70 years or more, and a logistic EuroSCORE of $20 \%$ or more. Patients with a life expectancy of 1 year or less, serum creatinine of $2.0 \mathrm{mg} / \mathrm{dL}$ or more, prior valve surgery, endocarditis during the past 18 months, recent myocardial infarction $(<30$ days), stroke during the prior 6 months, or clinically significant mitral regurgitation (>grade $2+4$ ) were excluded from the trial. Computed tomographic scans of the aorta and the fem- oral and iliac vessels were performed and reconstructed in 3 dimensions to evaluate patients for iliofemoral calcification, tortuosity, and access vessel diameter (femoral artery minimum inner diameter of $7.5 \mathrm{~mm}$ ). Mean logistic EuroSCORE for the study patients was $28 \% \pm 7 \%$. The majority $(71 \%)$ of patients were in New York Heart Association (NYHA) functional class III, $26 \%$ of patients were in NYHA class II, and 3\% were in NYHA class I (Figure 3). Preinterventional transthoracic echocardiography (TTE) revealed a mean aortic transvalvular gradient of $49 \pm 14 \mathrm{~mm} \mathrm{Hg}$ and an effective orifice area of $0.5 \pm 0.16 \mathrm{~cm}^{2}$ (Figure 4). Mean left ventricular ejection fraction was $53 \% \pm 15 \%$. The study was approved by the German National Committee and locally by the Ethics Committees of the Hamburg and Siegburg Boards of Physicians, and all patients provided written informed consent in accordance with the principles of the Declaration of Helsinki.

\section{Valve Implantation}

All procedures were performed using general anesthesia in a hybrid suite equipped for cardiologic interventions and cardiac operations. Transesophageal echocardiography (TEE) was performed throughout the procedure. All patients received acetylsalicylic acid (at least $100 \mathrm{mg}$ daily) and clopidogrel (300 mg loading dose, if patient was not currently receiving clopidogrel) and then $75 \mathrm{mg}$ daily 3 to 5 days before the procedure. Acetylsalicylic acid therapy was continued for life and an antiplatelet regimen was continued for at least 6 months after the procedure in all patients as per the study protocol. After surgical dissection of the right or left femoral artery, a weight-dependent bolus of 5000 to 10,000 units of heparin was administered intravenously to achieve an activated clotting time of more than 300 seconds. A pacemaker lead was placed in the right ventricle through the right jugular vein and tested for proper performance. A pigtail catheter was placed in the ascending aorta over a $6 \mathrm{~F}$ sheath through the contralateral femoral artery. The $22 \mathrm{~F}$ introducer sheath was placed carefully in the dissected femoral artery under fluoroscopic control. Balloon valvuloplasty was then performed with standard technique using rapid pacing (180-220 beats/min) and valvuloplasty balloons ranging from 18 $\mathrm{mm}$ to $28 \mathrm{~mm}$. Valvuloplasty was repeated until sufficient mobilization of the native leaflets was achieved and the mean pressure gradient dropped to $30 \mathrm{~mm} \mathrm{Hg}$ or less. In the first half of the study a median of 7 inflations occurred, whereas in the second half of the study a median of 5 inflations resulted in a typical gradient of $30 \mathrm{~mm} \mathrm{Hg}$ or less. A stiff wire was placed in the left ventricle through the stenotic native valve before the loaded delivery catheter was introduced and carefully advanced over the wire through the aorta. Once the device tracked through the aorta and crossed the native valve, the delivery catheter was pulled back and the deflated valve was unfolded in the left ventricle. After inflation of the ventricular ring with 50:50 saline/contrast, the pericardial leaflets were immediately competent and no additional rapid pacing was required. Adjusting the PFLs allowed the lower (ventricular) ring to be positioned and aligned in the native aortic annulus and the left ventricular outflow tract under fluoroscopic and echocardiographic visualization. Then, the upper (aortic) ring and the transverse tubes were inflated. This pushed the calcified native valve against the aortic root, thereby covering the native annulus. The prosthetic valve was then tested for correct subcoronary position, proper function and paravalvular leakage by TEE, fluoroscopy, and aortography. If necessary, repositioning was performed after partial deflation, or if needed the valve was completely removed and exchanged for a valve of a different size. When correct position and function were achieved, the saline/contrast mix was exchanged with a fluid polymer under constant pressure of 8 to 10 atmospheres. The full saline/polymer exchange occurred in a maximum of 10 minutes with the polymer firmly gelled in another 90 minutes and fully cured within 24 hours. During the exchange of the inflation media, the valve remained fully pressurized, competent, and in position. The PFLs were then detached from the upper ring and the delivery system removed. TEE and aortography were performed to reconfirm coronary flow, paravalvular and central leakages, transvalvular pressure gradient, and valve opening area. The femoral 


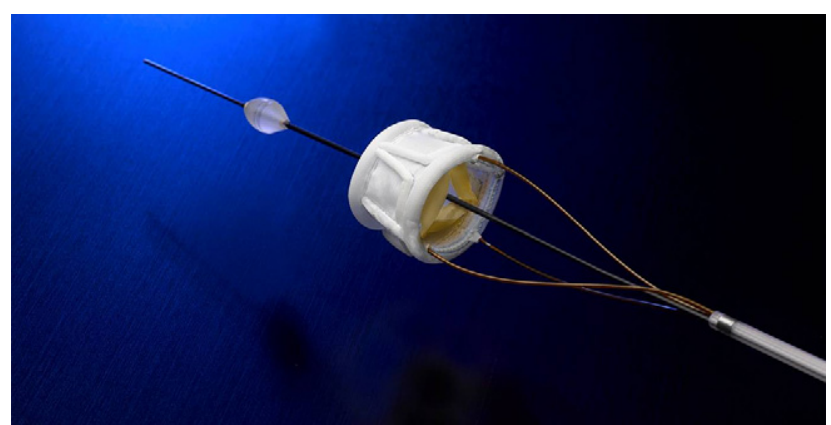

FIGURE 1. Expanded Direct Flow Medical aortic valve connected to positioning-and-fill lumina (PFLs).

artery was closed after removal of the introducer sheath by tying the purse-string sutures. Then the access site was closed surgically.

\section{Follow-up}

Patients underwent follow-up examinations including transthoracic echocardiography (TTE) and NYHA functional class status after 1 day, 30 days, and 6 months. To make accurate baseline and follow-up comparisons, we have reported all echocardiographic data from TTE.

\section{Statistics}

Continuous variables are presented by mean and interquartile range (IQR). Categorical variables are presented as counts and percentages. Ninety-five percent confidence intervals were calculated on the basis of the binomial distribution. Changes in continuous variables between baseline and at 30 days and 6 months were assessed by the Wilcoxon signed rank test. Comparisons between categorical variables were performed with the continuity-corrected $\chi^{2}$ test. These analyses used StatView 4.5 software package (Abacus Concepts, Inc, Berkeley, Calif). All statistical calculations were completed by an independent statistician. Authors had full access to the complete data set and take responsibility for its integrity. All authors have read and agreed to the manuscript as written.

\section{RESULTS}

\section{Procedure to Hospital Discharge}

Thirty-one patients were enrolled in the study on providing written informed consent before study-related diagnostic imaging. Diagnostic imaging was performed with TTE and 3-dimensional reconstruction of computed tomographic scans of the access site and native aortic valve.

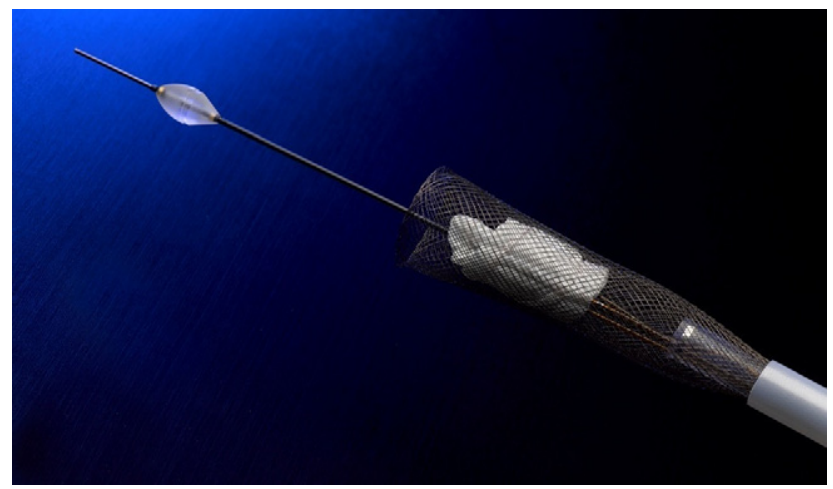

FIGURE 2. Direct Flow Medical aortic valve enfolded in nitinol retrieval basket.
TABLE 1. List and percentage of comorbidities of all patients (intention-to-treat group)

\begin{tabular}{lc}
\hline Baseline characteristics & $\%(\mathbf{n})$ \\
\hline Abdominal surgery & $29 \%(9)$ \\
Angina (stable) & $16 \%(5)$ \\
Arrhythmia (asymptomatic) & $39 \%(12)$ \\
Arrhythmia (symptomatic) & $7 \%(2)$ \\
CABG (prior) & $10 \%(3)$ \\
Cancer & $42 \%(13)$ \\
Carotid artery disease & $32 \%(10)$ \\
CHF (drug compensated) & $39 \%(12)$ \\
CHF (symptomatic) & $35 \%(11)$ \\
Chronic renal insufficiency & $39 \%(12)$ \\
COPD & $19 \%(6)$ \\
Coronary artery disease & $52 \%(16)$ \\
CVA & $13 \%(4)$ \\
Diabetes & $29 \%(9)$ \\
Hypertension & $74 \%(23)$ \\
MI $<6$ mo & $3 \%(1)$ \\
MI $>6$ mo & $7 \%(2)$ \\
Peripheral artery disease & $26 \%(8)$ \\
Prior PTCA & $26 \%(8)$ \\
TIA & $3 \%(1)$ \\
\hline CABG Co
\end{tabular}

$C A B G$, Coronary artery bypass grafting; $C H F$, congestive heart failure; $C O P D$, chronic obstructive pulmonary disease; $C V A$, cerebrovascular accident; $M I$, myocardial infarction; PTCA, percutaneous transluminal coronary angioplasty; TIA, transient ischemic attack.

All 31 patients were included in the intention-to-treat population. Figure 5 demonstrates the study flow of these patients.

In 9 patients, transfemoral implantation of the valve was not attempted or was not successful owing to the following reasons:

- Excessive tortuosity and calcification of the native vasculature prevented successful insertion of the catheter $(n=2)$. One of the patients was successfully treated with a 23-mm transapical Edwards SAPIEN prosthesis

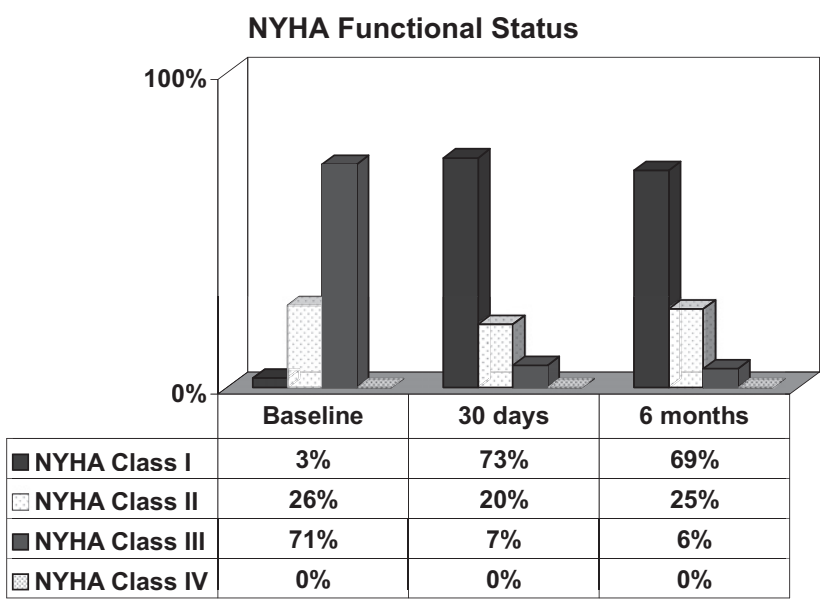

FIGURE 3. New York Heart Association (NYHA) functional class before and after valve implantation. 


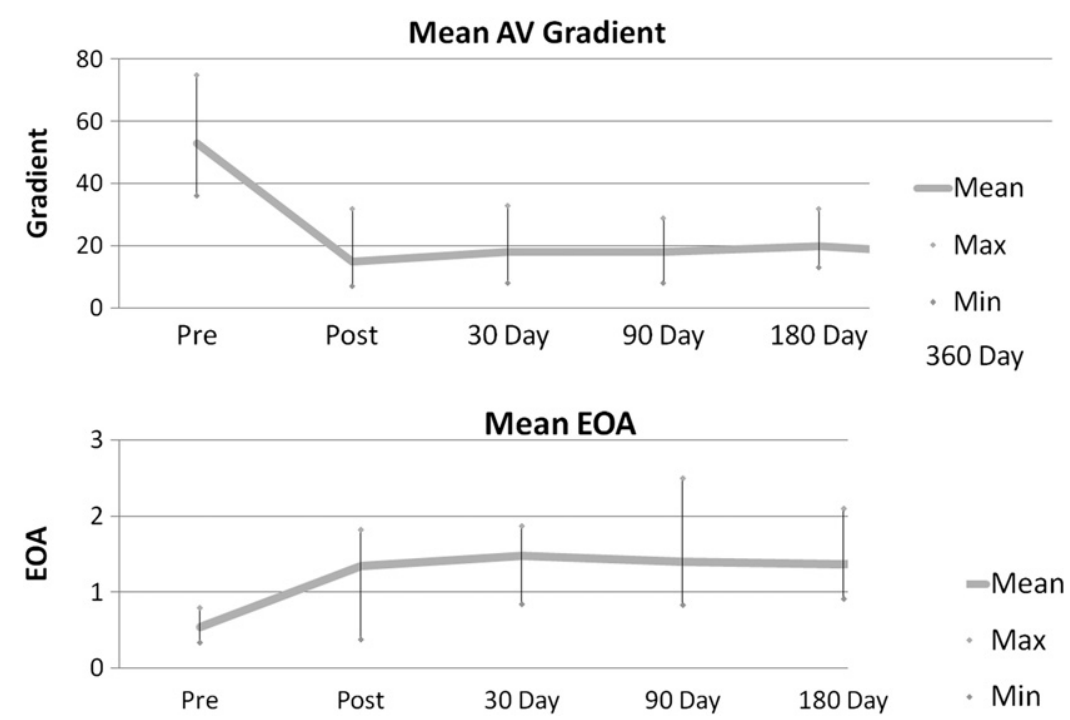

FIGURE 4. Mean transvalvular gradients $(\mathrm{mm} \mathrm{Hg})$ and mean effective orifice area $\left(\mathrm{cm}^{2}\right)(P=.001$ and $P=.002$ before and after intervention, respectively). $A V$, Aortic valve; $E O A$, effective orifice area.

4 months after the initial procedure. The other patient refused to undergo another procedure and was treated medically during the follow-up period.

- Functionally bicuspid aortic valves prevented successful ballooning of the native valve $(n=2)$. In the first patient, an attempt to implant a 23-mm device was unsuccessful owing to improper expansion of the cuff. The valve was successfully retrieved by the retrieval basket system and the patient underwent successful surgical aortic valve replacement 9 days later. In the second patient, no device implantation was attempted. Balloon valvuloplasty reduced the maximum aortic pressure gradient from 102 to $35 \mathrm{~mm} \mathrm{Hg}$. The patient was treated medically during the follow-up period of 6 months.

- Excessive calcification of the left ventricular outflow tract impeded correct positioning of the lower ring $(\mathrm{n}=1)$. This valve was successfully retrieved. The patient could only be treated medically during the follow-up period of 6 months.

- The annular diameter of the native valve was in excess of $27 \mathrm{~mm}$ and was underestimated in the preoperative computed tomographic scans. Implantation was not possible $(\mathrm{n}=1)$. This valve was successfully retrieved. The patient was given medical therapy and followed up for 1 month.

- Excessive calcification of the native leaflets hindered valve deployment $(n=1)$. This valve was successfully retrieved. The patient was also given medical therapy and followed up for 1 month.

- Septal dissection occurred during valvuloplasty $(\mathrm{n}=1)$. The patient died during the procedure before catheter insertion. This death was adjudicated by a Clinical Events Committee (CEC) to be procedurerelated but not device-related.

- Pulmonary embolism occurred during the procedure $(\mathrm{n}=1)$. The valve was successfully retrieved, but the patient never recovered. This death was also adjudicated by a CEC to be procedure-related but not device-related.

Those patients who did not receive the study valve were followed up out to 30 days after the procedure and then were considered to have completed the study. Six months of data are available for 7 of 9 patients.

Twenty-two patients underwent implantation of the Direct Flow Medical aortic valve (procedural success rate $71 \%$ ). Procedure time varied between the first 12 and the last 10 cases with respect to the learning curve. In the first 12 patients, mean procedure time was 192.8 minutes with an IQR of 87.5 minutes (start of cutdown to final closure). In the last 10 patients, mean procedure time was $174.7 \mathrm{~min}$ utes with an IQR of 117.0 (start of cutdown to final closure). Mean fluoroscopy time was 39.2 minutes. Transvalvular pressure gradients decreased from a mean of $50.2 \mathrm{~mm} \mathrm{Hg}$ (IQR $40.5-59 \mathrm{~mm} \mathrm{Hg}$ ) preoperatively to $14.9 \mathrm{~mm} \mathrm{Hg}$ (IQR $10.5-18.5 \mathrm{~mm} \mathrm{Hg})$ postoperatively $(P<.0001)$. The effective orifice area was increased from a mean of 0.55 $\mathrm{cm}^{2}$ (IQR $0.43-0.64 \mathrm{~cm}^{2}$ ) to $1.39 \mathrm{~cm}^{2}$ (IQR $1.21-1.7 \mathrm{~cm}^{2}$ ) over the same time period $(P<.0001)$. Paravalvular leakage and aortic regurgitation (AR) were limited with a maximum AR score of AR grade $1+/ 4$, with the exception of 1 patient who died on the day of the procedure of congestive heart failure (AR grade 2+/4).

Four major adverse cardiovascular and cerebral events (MACCEs) occurred in-hospital after successful valve 
ITT population

$n=31$

15 male; 16 female; mean age $84 \pm 4$ years

\section{Procedural Success Rate $71 \%$}

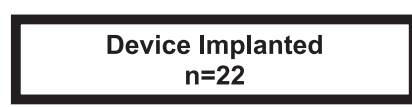

Surgical conversion $(n=2)$

( 1 sizing problem, 1 placement failure)
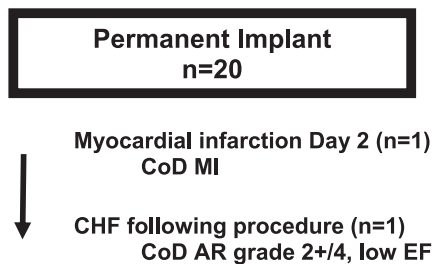

CoD AR grade $2+14$, low EF

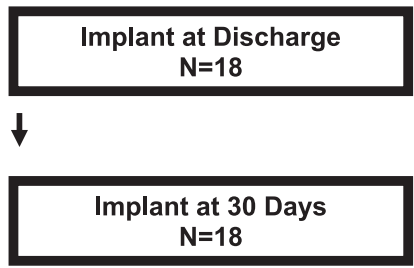

Non device related pneumonia Day $189(n=1)$ CoD

- Resp. failure

Death of unknown reason Day $142(n=1)$ CoD

possibly of cardiac reason

Implant at 6 Months

$\mathrm{N}=16$

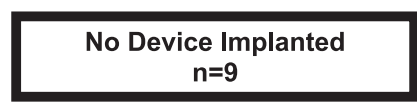

Iliac access $(n=2)$

Functionally bicuspid valve $(n=2)$

Excessive LVOT calcification $(n=3)$

Annular $\varnothing \uparrow \uparrow$, excessive calcification $(n=1)$

Excessive valvular calcification $(n=1)$

2 patients died

CoD: Septal rupture $(n=1)$

Pulmonary embolism $(n=1)$

FIGURE 5. Diagram illustrating study flow of intention-to-treat patient population. ITT, Intention to treat; $L V O T$, left ventricular outflow tract; $C o D$, cause of death; $M I$, myocardial infarction; $C H F$, congestive heart failure; $A R$, aortic regurgitation; $E F$, ejection fraction; Resp, respiratory.

implantation (MACCE rate 12.9\%; 4/31 patients): 1 patient with a history of chronic atrial fibrillation had a postprocedural stroke with dysarthria and weakness of the right arm at day 1 , another patient died on day 2 of inferior wall myocardial infarction, and 2 patients showed an increase in transvalvular gradients postoperatively and underwent surgical aortic valve replacement at day 2 and day 8, respectively. In those patients converted to surgery, gradient increase was attributed to an oversized valve with consecutive distortion of the upper ring in 1 patient and incorrect positioning of the properly sized valve in the second patient.

Other significant events included the following*: obstruction of the left coronary artery by a plaque shift from a highly calcified aortic root, which occurred after valve deployment. Stenting of the left main stem was performed immediately with good procedural result. Further, atrioventricular con-

\footnotetext{
* For the purpose of a thorough review, events are listed individually rather than by patient. One patient may account for more than one event discussed in this article.
}

duction blockage was seen in 3 patients and led to uncomplicated postinterventional pacemaker implantation. Of these 3 patients, 2 continue to require long-term pacing and the third did not require pacing after discharge.

In total, 18 patients were discharged from hospital with permanent implants. Of this population, 17 patients had an uneventful course and were discharged after a mean of 7 days (IQR 6-7 days). The patient who had the stroke was not immediately discharged but rather was sent to a rehabilitation unit for further care.

\section{Thirty-Day Follow-up}

Clinical follow-up was completed after 30 days on the discharged population. Survival at 30 days for the 18 patients who were discharged from the hospital with permanent implants was $100 \%$ and overall mortality of the intentionto-treat population at this time point was $12.9 \%$ (4 deaths in 31 patients). TTE revealed an increase in transvalvular gradient that was not clinically significant (mean gradient 
$19.4 \mathrm{~mm} \mathrm{Hg}$ vs $14.9 \mathrm{~mm} \mathrm{Hg} ; P=.007)$, whereas effective orifice area did not change significantly after the procedure $\left(1.46 \mathrm{~cm}^{2}\right.$ vs $\left.1.39 \mathrm{~cm}^{2} ; P=.8\right)$ (Figure 4). Paravalvular and intravalvular leaks were identical to the postprocedural examination (no leak in 11 patients; minor paravalvular leaks [AR grade 1/4] in 5 patients; minor central leaks [AR grade 1/4] in 2 patients). NYHA functional class improved significantly 30 days after valve implantation. The majority of patients $(73 \%)$ were in NYHA functional class I, $20 \%$ were in class II, and $7 \%$ in class III $(P=.015)$ (Figure 3).

\section{Six-Month Follow-up}

Six-month survival for the 18 patients who were discharged from the hospital with permanent implants was $89 \%$ ( 2 deaths in 18 patients). Overall 6-month survival of the intention-to-treat population was $80.6 \%$ (6 deaths in 31 patients).

One patient died during a routine hospital stay after spinal surgery as a result of acute respiratory failure after pneumonia 193 days after implantation. This death was adjudicated by the CEC as not related to the procedure or the study valve. A second patient died of an unknown cause while in rehabilitation 142 days after implantation. This death was considered by the CEC as possibly of cardiac origin although the final cause of death remains unclear.

TTE at 6 months revealed stable transvalvular gradients (mean gradient $19.8 \mathrm{~mm} \mathrm{Hg}$ vs $19.4 \mathrm{~mm} \mathrm{Hg}$ at 30 days) and an insignificant decrease in effective orifice areas $\left(1.30 \mathrm{~cm}^{2}\right.$ vs $1.46 \mathrm{~cm}^{2}$ at 30 days) (Figure 4). NYHA functional class remained improved at 6 months with $69 \%$ of patients in class I, $25 \%$ in class II, and $6 \%$ in class III (Figure 3 ). The majority of patients did not show any paravalvular leaks $(68.8 \%, 11 / 16$ patients). Only minor paravalvular leaks (AR grade 1/4) were found in 4 patients and a minor central leak (AR grade 2/4) in 1 patient.

\section{DISCUSSION}

Symptomatic aortic stenosis carries a poor prognosis. Surgical treatment by conventional aortic valve replacement using cardiopulmonary bypass and cardioplegic arrest has been established as the gold standard therapy with defined indications, low operative risk, and excellent long-term outcome. However, the proportion of patients with severe comorbidities and advanced age is rising and thus surgical risk may be deemed prohibitively high. For these patients, TAVI has become an alternative treatment option, and centers throughout Europe have rapidly adopted this new interventional technique.

After market approval, European centers have rapidly adopted the metallic stented TAVI devices (Edwards SAPIEN and Medtronic CoreValve). Data from ongoing registries of both devices reveal mortality rates of less than $10 \%$ in experienced centers. ${ }^{6-8}$ For both valve types, second- or third-generation devices are now available and have overcome the developmental stage of a first-in-man device.

As a favorable advantage over metallic stented valves, the Direct Flow Medical aortic valve offers the possibility of valve repositioning and valve retrieval during the procedure. Nevertheless, the device used in this study is a firstgeneration device and the data reported in this study reflect the first-in-man experience with this novel valve type. Therefore, procedural results should be compared with the first-generation results of the SAPIEN and CoreValve devices.

In 2006 Grube and associates ${ }^{9}$ reported on the first-in-man experience of the percutaneous implantation of the CoreValve prosthesis in 25 patients with a median logistic EuroSCORE of only $11.0 \%$. Device success and procedural success were achieved in $22(88 \%)$ and $21(84 \%)$ patients, respectively. Mortality was $20 \%$ (5/25 patients) before discharge and the MACCE rate was $32 \%$ ( $8 / 25$ patients).

In 2007 Webb and colleagues ${ }^{10}$ reported on the early experience with the Edwards SAPIEN prosthesis. Transfemoral valve implantation was attempted in 50 patients (EuroSCORE 28\%) with a procedural success rate of $86 \%$ ( $76 \%$ in the first 25 patients and $96 \%$ in the last 25 patients). Thirty-day mortality was $12 \%$ (6/50 patients), and procedure-related strokes occurred in 2 patients.

When compared with those results, the procedural success rate of the novel Direct Flow Medical aortic valve was lower $(71 \%, 22 / 31$ patients). This is possibly due to the lack of experience with transcatheter heart valve techniques during the initial stage of the study, as the Direct Flow Medical aortic valve is the first type of transcatheter aortic valve used at the University Heart Center, Hamburg. With current experience of more than 1000 TAVI cases at our center, a more refined patient selection process may have had a more positive impact on patient outcomes.

Nevertheless, the 30-day mortality rate of $12.9 \%$ (4/31 patients) compares favorably with data from first-in-man series of both CoreValve and Edwards SAPIEN prostheses. ${ }^{9,10}$ Preoperative risk assessment revealed a mean logistic EuroSCORE of $28 \% \pm 7 \%$ resulting from advanced age and relevant comorbidities in this high-risk patient population.

In addition, the rate of cerebrovascular complications has been very low with only one periprocedural stroke. Presumably, this is due to the high degree of flexibility of the delivery system and the olive-shaped tip facilitating a safe and atraumatic passage across the aortic arch and the native valve, even in patients with complex aortic calcifications.

An additional device-specific advantage of the Direct Flow Medical valve is its entirely subcoronary position, minimizing the risk of coronary ostial obstruction and compromised coronary perfusion.

In this stentless valve type, the number of balloon valvuloplasties and, accordingly, the number of rapid pacing episodes needed to create an adequate circular opening in the 
native annulus was higher than in metallic framed valve types. The importance of a circular opening is emphasized by 1 particular case in which a noncircular distortion of the upper ring resulted in an increased gradient that led to surgical aortic valve replacement 2 days after the procedure. A possible reason for the failure could be the lower radial force of this valve design as compared with stent-based valve types. Furthermore, in 2 patients valve implantation failed because of functionally bicuspid valves that did not respond sufficiently to balloon valvuloplasty even after multiple inflations. Bicuspid valves are now contraindicated for both of the commercially available metallic stented valves as well.

On the other hand, ventricular pacing is not necessary during the positioning process, allowing for controlled positioning, deployment, and even repositioning of the prosthesis under constant TEE monitoring on the beating heart. This stands in contrast to single-shot devices like the CoreValve and Edwards SAPIEN valves, which have only very limited options to correct malpositioning once the valve has been deployed.

Another important advantage of a nonmetallic valve design is that it effectively reduces paravalvular leakage. The majority of patients followed up to date do not show significant paravalvular or central leakages at 6 months. This compares favorably with published rates even of the latest generation of stent-based metallic valves. ${ }^{9-12}$

In contrast to commercially available stent-based metallic valves; the Direct Flow Medical aortic valve is repositionable and retrievable. In those patients in whom the implant could not be optimally positioned $(n=5)$, retrieval of the valve was safe. In patients in whom prosthesis size was miscalculated, the initial valve was easily retrieved and replaced by a properly sized implant. Therefore, retrieval complications owing to incorrect sizing or positioning of the valve did not occur.

In this safety and feasibility trial of the Direct Flow Medical aortic valve, the number of patients who could not be treated successfully demonstrates the need for proper patient selection and expresses the impact of the learning curve with this new technique. Patients with excessive calcification of the aortic valve or of the left ventricular outflow tract were unsuitable candidates for the implantation of a nonmetallic stentless valve prosthesis. Patients with moderate calcifica- tions may profit from the flexible design of the valve and the delivery system with regard to a lower rate of paravalvular leaks, a low stroke rate, and the possibility for valve repositioning and retrieval.

The results of this study cannot be transferred to patients of younger age and nonsignificant surgical risk profiles. Long-term results of this valve beyond 6 months must be observed before final statements about longevity and performance can be made. Increased patient experience and a careful patient selection process will help to define reliable morbidity and mortality rates for this novel device.

\section{References}

1. Iung B, Baron G, Butchart EG, Delahaye F, Gohlke-Bärwolf C, Levang OW, et al. A prospective survey of patients with valvular heart disease in Europe: The Euro Heart Survey on Valvular Heart Disease. Eur Heart J. 2003;13:1231-43.

2. Schofer J, Schlüter M, Treede H, Franzen O, Tübler T, Pascotto A, et al. Retrograde transarterial implantation of a nonmetallic aortic valve prosthesis in highsurgical-risk patients with severe aortic stenosis: a first-in-man feasibility and safety study. Circ Cardiovasc Intervent. 2008;2:126-33.

3. Bolling SF, Rogers JH, Babaliaros V, Piazza N, Takeda PA, Low RI, et al. Percutaneous aortic valve implantation utilizing a novel tissue valve: preclinical experience. EuroIntervention. 2008;4:148-53.

4. Low RI, Bolling SF, Yeo KK, Ebner A. Direct Flow Medical percutaneous aortic valve: proof of concept. EuroIntervention. 2008;4:256-61

5. Nashef SA, Roques F, Michel P, Gauducheau E, Lemeshow S, Salamon R. European System for Cardiac Operative Risk Evaluation (EuroSCORE). Eur J Cardiothorac Surg. 1999;16:9-13.

6. Zajarias A, Cribrier A. Outcomes and safety of percutaneous aortic valve replacement. J Am Coll Cardiol. 2009;53:1829-36.

7. Laborde JC. Transcatheter aortic valve implantation with the CoreValve ReValv ing Device. Presented at: Transcatheter Cardiovascular Therapeutics; October 12, 2008; Washington, DC.

8. Thomas M. Thirty-day results of the "Source registry": the European registry of transcatheter aortic valve implantation using the Edwards SAPIEN valve. Paper presented at: EuroPCR; May 20, 2009; Barcelona, Spain.

9. Grube E, Laborde J, Gerckens U, Felderhoff T, Sauren B, Buellesfeld L, et al. Percutaneous implantation of the CoreValve self-expanding valve prosthesis in high-risk patients with aortic valve disease-the Siegburg first-in-man study. Circulation. 2006;114:1616-24

10. Webb J, Pasupati S, Humphries K, Thompson C, Altwegg L, Moss R, et al. Percutaneous transarterial aortic valve replacement in selected high-risk patients with aortic stenosis. Circulation. 2007;116:755-63.

11. Vahanian A, Alfieri O, Al-Attar N, Antunes M, Bax J, Cormier B, et al. Transcath eter valve implantation for patients with aortic stenosis: a position statement from the European Association of Cardio-Thoracic Surgery (EACTS) and the European Society of Cardiology (ESC), in collaboration with the European Association of Percutaneous Cardiovascular Interventions (EAPCI). EuroIntervention. 2008;2: 193-9.

12. Grube E, Schuler G, Buellesfeld L, Gerckens U, Linke A, Wenaweser P, et al. Percutaneous aortic valve replacement for severe aortic stenosis in high-risk patients using the second- and current third-generation self-expanding CoreValve prosthesis: device success and 30-day clinical outcome. J Am Coll Cardiol. 2007;50: 69-76. 